\title{
Ultimate Population Size: Some Investigations Under Stable Population Theory
}

\author{
K.N.S. Yadava ${ }^{1, *}$, Shruti $^{2}$, J.Pandey ${ }^{1}$ \\ ${ }^{1}$ Department of Statistics, Banaras Hindu University, Varanasi, India \\ ${ }^{2}$ Uttar Pradesh Rajarshi Tandon Open University, India \\ *Corresponding Author: knsy_bhu@yahoo.com
}

Copyright $@ 2014$ Horizon Research Publishing All rights reserved.

\begin{abstract}
Some models have been proposed for the projection of future size of population for short and long terms under the stability conditions with changed regime of fertility schedule. The main aim of this paper is to see the size of population if fertility is curtailed up to the level of replacement, especially in developing countries. Models have been illustrated taking a set of real and hypothetical data consistent with the current demographic scenario of India. It was found that the proposed models are the extended forms of the models developed by the previous researchers and the projected population are more or less consistent with them.
\end{abstract}

Keywords Stability Conditions, Ultimate Population, Fertility Schedule, Models, Replacement Level

\section{Introduction}

In a stable population theory when population growth rate becomes zero, the population size becomes fixed and such population is known as stationary population or a population at the replacement level or an ultimate population. Several ways of reduction in fertility schedule have been proposed to obtain population size under stable and stationary conditions (Frauenthal [3]; Keyfitz [4]; Kulkarni [7]; Mitra [9]; Pathak and Ram [10]; Ryder [11]; Singh et.al. [12] and [13]; Yadava [14]; Yadava et.al. [15] and [16]). Keyfitz [4] considered an abrupt change in age specific fertility rate say in $m(x, t)$ at time $\mathrm{t}$ as $\mathrm{m}(\mathrm{x}) / \mathrm{Ro}$, where Ro is the net reproduction rate. The state of stationary is supposed to yield a new NRR as unity (see Figure 1), but it is not feasible due to population momentum built up in the past. That is the size of ultimate population would be larger than the expected one due to the existence of a high fertility rate built up by the past age structure (Keyfitz [4] and Kulkarni [7] (see Figure 2). Nevertheless, the size of population does not depend only on the way of reduction in fertility but also on the several other existing parameters of the population.

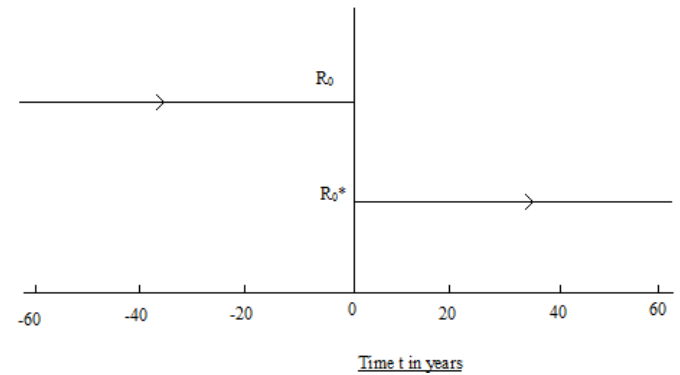

Figure 1. Time history of net reproduction rate under an abrupt change in fertility schedule.

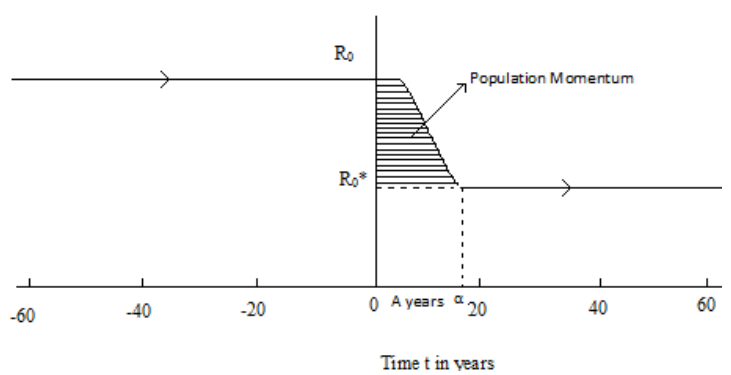

Figure 2. Time history of net reproduction rate under a gradual change in fertility schedule.

Keyfitz [4] has shown that when the age specific fertility rate $m(x)$ is replaced by $m(x) / R o$, such that new NRR, say Ro*, becomes unity, the ultimate population size is given by

$$
\frac{b e_{0}^{0}}{\mu r}\left(1-\frac{1}{R_{0}}\right)
$$

where, $b$ : the present initial annual number of births; $\mathrm{e}_{0}^{0}$ : expectation of life at birth; $\mu$ : mean age of child bearing in stationary population; Ro : the present net reproduction rate and $r$ :the present annual rate of natural increase

However, as mentioned above, an abrupt change in fertility schedule is not realistic even not feasible. Moreover, due to momentum, the population tends to increase for some time as shown in Figure 2 Accounting such type of decay if it for A years ( $A$ is less than or equal to the lower limit of the reproductive period), Ryder [11] made an adjustment to Keyfitz's model (1) and according to him the ultimate population size becomes,. 


$$
\frac{b e_{0}^{0}}{\mu r}\left(1-\frac{1}{R_{0}}\right) e^{r \frac{A}{2}}
$$

Further if fertility schedule $\mathrm{m}(\mathrm{x})$ declines exponentially during the transition period of A years, an approximate formula for ultimate population size given by Coale [1] is:

$$
\begin{gathered}
b e_{0}^{0} e^{r\left(A-T+\frac{3}{32} \frac{T^{2}}{A}\right)} \quad \text { if } A<\frac{3}{4} T \\
b e_{0}^{0} e^{\frac{r}{2}(A-T)} \quad \text { if } \quad A \geq \frac{3}{4} T
\end{gathered}
$$

Where $T \cong \frac{\ln R_{0}}{r}$ is the mean length of generation.

Usually $T$ varies from country to country ranging between 27 to 29 years. On an average, if $T=28$ years, then by the Coale's model one can project population for a period more than the lower limit of the reproductive value. Kulkarni [7] has proposed two types of reduction pattern in fertility schedule, namely exponential and linear if time to reach the replacement level is less than or equal to the lower limit of the reproductive period. According to him, if $\mathrm{m}(\mathrm{x})$ declines exponentially as :

$$
m(x, t)= \begin{cases}m(x) & ; t<0 \\ m(x) e^{k t} & ; 0 \leq t<A \\ \frac{m(x)}{R_{0}} & ; A \leq t \text { and } A \leq \alpha\end{cases}
$$

Where $k=-\ln R o / A$, so that $e^{k A}=1 / R o$ and $\alpha$ is the lower limit of the reproductive period. Under such reduction, the ultimate population size becomes (Kulkarni [7]),

$$
\frac{b e_{0}^{0}}{\mu r}\left(\frac{T}{T-A}\right)\left(1-\frac{e^{r} A}{R_{0}}\right) \text { for } A \leq \alpha
$$

and when $m(x)$ declines linearly as :

$$
m(x, t)=\left\{\begin{array}{cl}
m(x) & ; t<0 \\
m(x)\left(\frac{A-t}{A}\right)+\frac{m(x)}{R_{0}}\left(\frac{t}{A}\right) & ; 0 \leq t<A \\
\frac{m(x)}{R_{0}} & ; A \leq t \quad \text { and } A \leq \alpha
\end{array}\right.
$$

then the ultimate population size becomes (Kulkarni [7]),

$$
\frac{b e_{0}^{0}}{\mu r^{2} A}\left(1-\frac{1}{R_{0}}\right)\left(e^{r A}-1\right) \quad \text { for } \quad A \leq \alpha
$$

It should, however, be noted that for a general value of $A$, it is very difficult to find an exact formula for the ultimate population size. All the above formulae have been derived under the assumption that a constant proportion of decline occurs in the age specific fertility schedule $m(x, t)$ at time $\mathrm{t}$ at all the ages of the reproductive period which may not be reasonable as well as practicable for a society. An age dependent reduction in fertility schedule would be more reasonable and accordingly Mitra [9] suggested a reduction in fertility to replacement level that would be more for older women than the younger ones i.e. age specific fertility rate $m(x)$ be replaced by $m^{*}(x)=e^{-r x} m(x)$ rather than $\frac{m(x)}{R_{0}}$. Taking such a change in fertility schedule, Kumar [6] has derived expressions for the ultimate population size, allowing exponential and linear decay as proposed by Kulkarni [7] for A years $(A \leq \alpha)$ (see equations (4) and (6)). Allowing a decay of fertility to replacement level as a mixture of exponential for the first $A_{1}$ years and linear between $A_{1}$ to $A_{2}$ years $\left(A_{1}<A_{2} \leq A \leq \alpha\right)$, Yadava et.al.[17] has also derived some formulae for the size of ultimate population under $m^{*(x)}=m(x) e^{-r x}$.

However, it is expected that at the start of any family welfare activity, programme goes slowly and picked up its speed at some later stage. Thus, a linear type of reduction in fertility schedule would be more useful at the beginning and then exponential decline at the later stage. This approach has also been taken into account in this study and models are derived accordingly. For simplicity, formulae developed in this paper dealt only with one sex i.e. the female sex. The male population can be obtained as

Male population $=($ Female population $) *[($ Life expectancy for male $) /($ Life expectancy for female $)] *$ sex-ratio at birth

\section{Derivation of Models for Ultimate Population}

Lotka [8] has given a renewal equation for female births $B(t)$, at time $t$ for a population which is closed to migration, as follows: 
for $t>0$

$$
\begin{aligned}
B(t)=\int_{t}^{\infty} 1(x-t) \frac{p(x)}{p(x-t)} m(x, t) d x+\int_{0}^{t} B(t & -x) p(x) m(x, t) d x \\
& =G(t)+\int_{0}^{t} B(t-x) p(x) m(x, t) d x
\end{aligned}
$$

where $l(x)$ is the age distribution of females at time $t=0 ; p(x)$ is the survival function of a female up to age $x ; m(x) d x$ is the probability that a female of age $x$ will bear a female child during $\mathrm{dx}$ period of her life.

where $G(t)$ is the trajectory of births to women born before time zero (i.e. before start of the reduction in fertility) and the integral term gives births to women born after time zero i.e. during the transition period of fertility reduction.

If the base line population is stable then, we have

$$
l(x)=b e^{-r x} p(x)
$$

where $b$ is the instantaneous birth rate at $t=0$. Thus from equation (8) and (9), we get

$$
B(t)=b e^{r t} \int_{t}^{\infty} e^{-r \alpha} p(x) m(x, t) d x+\int_{0}^{t} B(t-x) p(x) m(x, t) d x
$$

For $t>0$

For $t \leq \alpha$, equation (10) reduces to

$$
\begin{aligned}
B(t)=G(t)=b e^{r t} \int_{t}^{\infty} e^{-r \alpha} p(x) & m(x, t) d x \\
& (\text { since } m(x, t)=0 \text { for } t \leq \alpha)
\end{aligned}
$$

Because $\int_{t}^{\infty} e^{-r x} p(x) m(x, t) d x=1$ for $t \leq \alpha$ is the fundamental equation for the intrinsic rate of increase $\mathrm{r}$. The equation (11) gives the birth trajectory at any time $t(t \leq \alpha)$ obtained by Frauenthal [3].

If all the changes in fertility schedule takes during $(o \alpha)$ and after $\alpha$, fertility remains constant i.e. $m(x, t)=m(x)$ for $t>\alpha$, thenthe renewal equation reduces to

$$
B(t)=G(t)=\int_{t}^{\infty} B(t-x) p(x) m(x, t) d x
$$

and the population ultimately reaches the stationary state with size (Keyfitz [4])

$$
K(\infty)=B(\infty) e_{0}^{0}=\frac{\int_{0}^{\beta} G(t) d t}{\mu} e_{0}^{0}
$$

where $G(t)$ is defined as,

$$
f(x)=\left\{\begin{array}{cc}
\int_{\alpha}^{\beta} B(t-x) p(x) m(x, t) d x & ; 0<t \leq \alpha \\
\int_{\alpha}^{\beta} B(t-x) p(x) m(x, t) d x & ; \alpha<t \leq \beta \\
0 & ; t>\beta
\end{array}\right.
$$

For an initially stable population,

$$
m(x, t)=m(x) \text { for } t>A
$$

and

$$
B(t-x)=B_{0} e^{r(t-x)}
$$

Further, if change in fertility is completed by A years $(A \leq \alpha)$, then

$G(t)$ can be written as

$$
G(t)= \begin{cases}\int_{\alpha}^{\beta} b e^{r(t-x)} p(x) m(x) d x & ; t \leq A \\ \int_{\alpha}^{\beta} b e^{r(t-x)} p(x) m(x) d x & ; A<t \leq \alpha \\ \int_{t}^{\beta} b e^{r(t-x)} p(x) m(x) d x & ; \alpha<t \leq A\end{cases}
$$

(letting $\mathrm{B}_{0}=\mathrm{b}$ )

Now taking the different way of reduction in fertility schedule $\mathrm{m}(\mathrm{x})$ and hence evaluating the value of $G(t)$, ultimate population size are projected by putting its values in equation (13).

\section{The Proposed Models}




\subsection{Mitra Form of Reduction in Fertility Schedule:}

As mentioned in the introductory section, if we take Mitra's form of reduction in $m(x)$ i.e. $m(x, t)=m(x) e^{-r x}$ (Mitra [9]), then an age dependent equation (6) becomes

$$
m(x, t)= \begin{cases}m(x) & ; t<0 \\ \frac{m(x)\left(A_{2}-t\right)}{A_{2}}+m(x)\left(\frac{t}{A_{2}}\right) e^{-r x} & ; 0<t<A_{1} \\ m(x) e^{k t} & ; A_{1}<t<A_{2} \\ m(x) e^{-r x} & ; A_{2}<t \leq \alpha\end{cases}
$$

where $k$ is a small positive integer

Now the value of $G(t)$ is,

$$
f(x)= \begin{cases}b e^{r t} \frac{\left(A_{2}-t\right)}{A_{2}}+\left(\frac{t}{A_{2}}\right) \int_{\alpha}^{\beta} e^{-2 r x} p(x) m(x) d x & ; t<A_{1} \\ b e^{(r+k) t} & ; A_{1} \leq t \leq A_{2} \\ b e^{r t} \int_{\alpha}^{\beta} e^{-2 r x} p(x) m(x) d x & ; A_{2}<t \leq \alpha \\ b e^{r t} \int_{\alpha}^{\beta} e^{-2 r x} p(x) m(x) d x & ; \alpha \leq t \leq \beta\end{cases}
$$

It is very difficult to evaluate the integrals like

$$
\int_{\alpha}^{\beta} e^{-2 r x} p(x) m(x) d x \text { or } \int_{t}^{\beta} e^{-2 r x} p(x) m(x) d x
$$

unless some functional form of the maternity function say,

$$
\phi(x)=m(x) p(x)
$$

is assumed. Mitra [9] assumed the nature of the maternity function as normal distribution and accordingly

Therefore,

$$
\begin{gathered}
\int_{\alpha}^{\beta} e^{-2 r x} p(x) m(x) d x \cong \frac{e^{r^{2} \sigma^{2}}}{R_{0}} \\
\int_{0}^{\beta} G(t) d t=\int_{0}^{A_{1}}\left[b e^{r t} \frac{\left(A_{2}-t\right)}{A_{2}}+\left(\frac{t}{A_{2}}\right) \frac{e^{r^{2} \sigma^{2}}}{R_{0}}\right] d t+\int_{A_{1}}^{A_{2}} b e^{(r+k) t} d t \\
+\int_{A_{2}}^{\alpha} b e^{r t} \frac{e^{r^{2} \sigma^{2}}}{R_{0}} d t+\int_{\alpha}^{\beta} b e^{r t}\left(\int_{t}^{\beta} e^{-2 r x} p(x) m(x) d x\right) d t
\end{gathered}
$$

and the size of population is,

$$
K(\infty)=\frac{b e_{0}^{0}}{\mu r}\left\{\left(e^{r A_{1}}-\frac{e^{r A_{2}+r^{2} \sigma^{2}}}{R_{0}}\right)-\left(\frac{A_{1} e^{r A_{1}}}{A_{2}}-\frac{e^{r A_{1}}}{A_{2} r}+\frac{1}{A_{2} r}\right)\left(1-\frac{e^{r^{2} \sigma^{2}}}{R_{0}}\right)+\frac{e^{(r+k) A_{2}}}{1+\frac{k}{r}}-\frac{e^{(r+k) A_{1}}}{1+\frac{k}{r}}\right\}
$$

Further if

$$
e^{k A_{2}}=\frac{1}{R_{0}} \text { or } k=-\ln R_{0} / A_{2} \text { and } r=\ln R_{0} / T
$$

then,

$$
\int_{0}^{\beta} G(t) d t=\frac{b}{r}\left\{\left(e^{r A_{1}}-\frac{e^{r A_{2}+r^{2} \sigma^{2}}}{R_{0}}\right)-\left(\frac{A_{1} e^{r A_{1}}}{A_{2}}-\frac{e^{r A_{1}}}{A_{2} r}+\frac{1}{A_{2} r}\right)\left(1-\frac{e^{r^{2} \sigma^{2}}}{R_{0}}\right)+\left(e^{r A_{1}-\frac{r T A_{1}}{A_{2}}}-\frac{e^{r A_{2}+r^{2} \sigma^{2}}}{R_{0}}\right)\left(\frac{A_{2}}{A_{2}-T}\right)\right\}
$$

which yield the ultimate population size as

$$
K(\infty)=\frac{b e_{0}^{0}}{\mu r}\left\{\left(e^{r A_{1}}-\frac{e^{r A_{2}+r^{2} \sigma^{2}}}{R_{0}}\right)-\left(\frac{A_{1} e^{r A_{1}}}{A_{2}}-\frac{e^{r A_{1}}}{A_{2} r}+\frac{1}{A_{2} r}\right)\left(1-\frac{e^{r^{2} \sigma^{2}}}{R_{0}}\right)+\left(e^{r A_{1}-\frac{r T A_{1}}{A_{2}}}-\frac{e^{r A_{2}+r^{2} \sigma^{2}}}{R_{0}}\right)\left(\frac{A_{2}}{A_{2}-T}\right)\right\}
$$

(Model M1)

\subsubsection{Some Special Cases}

(i) If $A_{1}=A_{2}=A$ (i.e. reduction in fertility schedule follows the same pattern up to the A years, then the ultimate 
population size from equation (20) becomes

$$
K(\infty)=\frac{b e_{0}^{0}}{\mu r}\left[\left(1-\frac{e^{r^{2} \sigma^{2}}}{R_{0}}\right)\left(\frac{e^{r A}-1}{r A}\right)-\frac{1}{R_{0}}\left(1-e^{r^{2} \sigma^{2}}\right)\left(\frac{A}{A-T}\right)\right]
$$

(Model M11)

(ii) Further, for small $r$,

the size of population is,

$$
K(\infty)=\frac{b e_{0}^{0}}{\mu r}\left(e^{r A}-1\right)\left(1-\frac{e^{r^{2} \sigma^{2}}}{R_{0}}\right)
$$

( Model M12)

The model M12 is same as Kumar [6] under a linear change in fertility schedule.

(iii) if $A_{1}=0$ and $A_{2}=A$ then,

the size of ultimate population is

$$
K(\infty)=\frac{b e_{0}^{0}}{\mu r}\left(1-\frac{e^{r A_{2}+r^{2} \sigma^{2}}}{R_{0}}\right)\left(\frac{T}{T-A}\right)
$$

(Model M13)

This model M13 is same as Kumar [6] under an exponential change in fertility schedule.

(iv) Further, for small $r$,

$$
e^{r^{2} \sigma^{2}} \cong 1
$$

and then models M12 and M13 reduce to Kulkarni's [7] under exponential and linear change in fertility schedule respectively.

\subsection{A Mixture of Abrupt and Gradual Change in Fertility Schedule:}

If we fix a reduction in fertility schedule in between Keyfitz's abrupt change and Kulkarni's gradual change, then a formula for ultimate population size is derived as follows. In such a situation

$$
\begin{aligned}
& m(x, t)= \\
& \begin{cases}m(x) & ; t<0 \\
m(x) e^{-k t}\left(1-\frac{t}{A}\right)+\frac{m(x)}{R_{0}}(t / A) e^{-k t} & ; 0<t<A \\
\frac{m(x)}{R_{0}} & ; t \leq \alpha\end{cases}
\end{aligned}
$$

where $\mathrm{k}$ is a small positive integer.

Then $G(t)$ is,

$$
m(x, t)= \begin{cases}\int_{\alpha}^{\beta} b e^{r(t-x)}\left[m(x) e^{-k t}\left(1-\frac{t}{A}\right)+\frac{m(x)}{R_{0}}\left(\frac{t}{A}\right) e^{-k t}\right] p(x) d & ; 0<t<A \\ \int_{\alpha}^{\beta} b e^{r(t-x)}\left[\frac{m(x)}{R_{0}}\right] \frac{m(x)}{R_{0}} & ; A<t \leq \alpha \\ \int_{t}^{\beta} b e^{r(t-x)}\left[\frac{m(x)}{R_{0}}\right] \frac{m(x)}{R_{0}} & ; \alpha \leq t \leq \beta\end{cases}
$$

Therefore,

$$
\int_{0}^{\beta} G(t) d t=\int_{0}^{A} b e^{r t}\left[e^{-k t}\left(1-\frac{t}{A}\right)+\left(\frac{t e^{k t}}{A R_{0}}\right)\right] d t+\int_{A}^{\alpha} \frac{b e^{r t}}{R_{0}} d t \quad+\int_{\alpha}^{\beta}\left[\frac{b e^{r t}}{R_{0}} \int_{\alpha}^{\beta} e^{-r x} p(x) m(x) d x\right] d t
$$

and the ultimate population size is

$$
K(\infty)=\frac{b e_{0}^{0}}{\mu r}\left[\left\{e^{r^{*} A}-1\right\}-\frac{1}{A}\left\{A e^{r^{*} A}-\frac{e^{r^{*} A}}{r^{*}}+\frac{1}{r^{*}}\right\}\left(1-\frac{1}{R_{0}}\right)+\frac{r^{*}}{r}\left(1-\frac{e^{r A}}{R_{0}}\right)\right]
$$

where $r-k=r^{*}$ 
if $k=0$, then model $\mathrm{M} 2$ reduces to

$$
K(\infty)=\frac{b e_{0}^{0}}{\mu A r^{2}}\left(e^{r A}-1\right)\left(1-\frac{1}{R_{0}}\right)
$$

(Model M21)

Kulkarni's [7] model under a linear change in fertility schedule.

\subsection{An Uniform Reduction in Fertility Schedule:}

If we assume an uniform decline in $m(x)$ in $(0, A)$, such that

$$
m(x, t)= \begin{cases}m(x) & ; t<0 \\ \frac{m(x)}{R_{0}} R_{0}^{*} & ; 0<t<A_{1} \\ \frac{m(x)}{R_{0}} & ; A_{1}<t<A_{2}\end{cases}
$$

Then,

$$
\begin{aligned}
\int_{0}^{\beta} G(t) d t=\int_{0}^{A} b e^{r t} \frac{R_{0}^{*}}{R_{0}} d t+\int_{A}^{\alpha} \frac{b e^{r t}}{R_{0}} d t & +\int_{\alpha}^{\beta}\left[\frac{b e^{r t}}{R_{0}} \int_{t}^{\beta} e^{-r x} p(x) m(x) d x\right] d t \\
& =\frac{b}{r}\left[\left(\frac{R_{0}^{*}}{R_{0}}-\frac{1}{R_{0}}\right) e^{r A}+\left(1-\frac{R_{0}^{*}}{R_{0}}\right)\right] \quad \text { for } \quad A \leq \alpha
\end{aligned}
$$

and the ultimate population size is

$$
K(\infty)=\frac{b e_{0}^{0}}{\mu r}\left[\left(\frac{R_{0}^{*}}{R_{0}}-\frac{1}{R_{0}}\right) e^{r A}+\left(1-\frac{R_{0}^{*}}{R_{0}}\right)\right]
$$

(Model M3)

If $R_{0}^{*}=1$, then the size of population is

$$
K(\infty)=\frac{e_{0}^{0}}{\mu} \frac{b}{r}\left(1-\frac{1}{R_{0}}\right)
$$

(Model M31)

a model given by Keyfitz [4].

\subsection{A Linear Reduction in Fertility Schedule:}

Kulkarni [7] proposed a linear change in $\mathrm{m}(\mathrm{x})$ (see equation (24)). Under such a linear change in fertility schedule, the value of $G(t)$ is

$$
G(t)= \begin{cases}b e^{r t}\left\{\left(1-\frac{t}{A}\right)+\frac{1}{R_{0}}\left(\frac{t}{A}\right)\right\} & ; t \leq A \\ \frac{b e^{r t}}{R_{0}} & ; A<t \leq \alpha \\ \frac{b e^{r t}}{R_{0}} \int_{t}^{\beta} e^{-r x} p(x) m(x) d x & ; \alpha<t \leq \beta\end{cases}
$$

If Yadava et.al. [17] approximation in the evaluation of complex integrals are taken into account, then $G(t)$ becomes

$$
G(t)= \begin{cases}b e^{r t}\left\{\left(1-\frac{t}{A}\right)+\frac{1}{R_{0}}\left(\frac{t}{A}\right)\right\} & ; t \leq A \\ \frac{b e^{r t}}{R_{0}} & ; A<t \leq \alpha \\ \frac{b e^{r t}}{R_{0}}\left(\frac{\beta-t}{\beta-\alpha}\right) & ; \alpha<t \leq \beta\end{cases}
$$

[where $\int_{t}^{\beta} e^{-r x} p(x) m(x) d x \cong \frac{\beta-t}{\beta-\alpha}$ (Yadava,et. al. [17])

Therefore

$$
\int_{0}^{\beta} G(t) d t=\int_{0}^{A} b e^{r t}\left\{\left(1-\frac{t}{A}\right)+\frac{1}{R_{0}}\left(\frac{t}{A}\right)\right\} d t+\int_{A}^{\alpha} \frac{b e^{r t}}{R_{0}} d t+\int_{\alpha}^{\beta} \frac{b e^{r t}}{R_{0}}\left(\frac{\beta-t}{\beta-\alpha}\right) d t
$$




$$
=X+Y+Z \quad \text { (say) }
$$

Where,

$$
\begin{gathered}
X=\int_{0}^{A} b e^{r t}\left\{\left(1-\frac{t}{A}\right)+\frac{1}{R_{0}}\left(\frac{t}{A}\right)\right\} d t \\
=b\left[\frac{e^{r A}-1}{r}-\frac{e^{r A}}{r}+\frac{e^{r A}}{A r^{2}}-\frac{1}{A r^{2}}+\frac{e^{r A}}{r R_{0}}-\frac{e^{r A}}{A R_{0} r^{2}}+\frac{1}{A R_{0} r^{2}}\right] \\
Y=\int_{A}^{\alpha} \frac{b e^{r t}}{R_{0}} d t=\frac{b}{r R_{0}}\left(e^{r \alpha}-e^{r A}\right) \\
Z=\int_{\alpha}^{\beta} \frac{b e^{r t}}{R_{0}}\left(\frac{\beta-t}{\beta-\alpha}\right) d t=\frac{b}{r R_{0}(\beta-\alpha)}\left[\left(\alpha e^{\alpha r}-\beta e^{\alpha r}\right)+\frac{1}{r}\left(e^{\beta r}-e^{\alpha r}\right)\right]
\end{gathered}
$$

After putting all these values in (34), we get

$$
\begin{array}{r}
\int_{0}^{\beta} G(t) d t=\frac{b}{r}\left[\left\{\frac{1}{A r}\left(e^{r A}-1\right)\right.\right. \\
\left.\left.\left(1-\frac{1}{R_{0}}\right)\right\}+\frac{e^{r a}}{R_{0}}-1\right]+\frac{b}{r}\left[\frac{1}{R_{0}(\beta-\alpha)}\left\{\left(\alpha e^{r a}-\beta e^{r a}\right)\right\}+\frac{1}{r}\left(e^{r \beta}-e^{r a}\right)\right] \\
=\frac{b}{A r^{2}}\left(e^{r A}-1\right)\left(1-\frac{1}{R_{0}}\right)+\frac{b e^{r a}}{r R_{0}}-\frac{b}{r}+\frac{b}{r R_{0}}\left[\frac{1}{r(\beta-\alpha)}\left(e^{r \beta}-e^{r a}\right)-e^{r a}\right]
\end{array}
$$

and hence the ultimate size of population as,

$$
K(\infty)=\frac{b e_{0}^{0}}{\mu r}\left[\frac{1}{A r}\left(e^{r A}-1\right)\left(1-\frac{1}{R_{0}}\right)+\left(\frac{e^{r a}}{R_{0}}-1\right)+\frac{1}{R_{0}}\left[\frac{1}{r(\beta-\alpha)}\left(e^{r \beta}-e^{r a}\right)-e^{r a}\right]\right]
$$

\subsection{A Simplified Model:}

It should, however, be noted that all the above models require data for many parameters to predict the size of the population. Some very specific models may be approximated as follows:

Let us take the example of model M13 from equation (23), where the ultimate population size is

$$
K(\infty)=\frac{b e_{0}^{0}}{\mu r}\left[\left(1-\frac{e^{r A}}{R_{0}}\right)\left(\frac{T}{T-A}\right)\right]
$$

(since $e^{r^{2} \sigma^{2}} \cong 1$, for a small value of $r$ )

(Since $\left.e^{r T} \cong R_{0}\right)$

$$
K(\infty)=\frac{b e_{0}^{0}}{\mu r}\left[\left(1-e^{r(A-T)}\right)\left(\frac{T}{T-A}\right)\right]
$$

$$
\begin{aligned}
& \cong \frac{b e_{0}^{0}}{\mu r}\left[\{r(T-A)\}\left(\frac{T}{T-A}\right)\right] \\
& \quad \cong \frac{b e_{0}^{0}}{\mu r}[\{r T\}] \\
& \left.\quad \cong b e_{0}^{0} \text { as } \mu \cong T \quad \text { (Coale }[1]\right)
\end{aligned}
$$

(Model M5)

Thus, the size of an ultimate population is $b_{0}{ }^{0}$ times the initial population. That is if only two parameters viz., birth rate and expectation of life at birth are available for a country/area, ultimate population can be projected for that country. By projecting population from equation (38), an extent of bias involved there can also be illustrated.

\subsection{Ultimate Population Size : Application of the Reproductive Value}

Keyfitz [5] has outlined that if current age specific fertility rate $m(a)$ is replaced by $m^{*}(a)$ such that $1<R_{0}^{*}<R_{0}$ as 


$$
m^{*}(a)=m(a) \frac{R_{0}^{*}}{R_{0}}
$$

(here $m(a)=m(x)$; both ' $a$ ' and ' $x$ ' represent age of a female during the reproductive period)

Then the population at time $t$ is,

$$
\frac{1}{K b^{*}} \int_{0}^{\beta} e^{r^{*} t} l(x) v^{*}(x) d x
$$

times the initial population.

Where,

$$
l(x)=b e^{-r x} p(x), \quad v^{*}(x)=\frac{1}{e^{-r^{*} x} p(x)} \int_{x}^{\beta} e^{-r^{*} a} p(a) m^{*}(a) d a
$$

and $K$ is the mean age of child bearing in the stable population. Further, if we assume an increase in survival function due to decreasing mortality rate in the survival function $p(a)$ as $p^{*}(a)$ i.e

$$
p^{*}(a)=p(a) e^{k a}
$$

where $k$ is a small positive integer.

Then after putting all these in equation (35), we have

$$
\begin{gathered}
\frac{1}{K b^{*}} \int_{0}^{\beta} e^{r^{*} t}\left(b e^{-r x} p(x)\right)\left(\frac{1}{e^{-r^{*} x} p(x)} \int_{x}^{\beta} e^{-r^{*} a} p(a) m^{*}(a) d a\right) d x \\
=\frac{b e^{r^{*} t}}{K b^{*}} \int_{0}^{\beta}\left(e^{-\left(r-r^{*}\right) x}\right) \\
\left(\int_{x}^{\beta} e^{-r^{*} a}\left(m(a) \frac{R_{0}^{*}}{R_{0}}\right)\left(p(a) e^{k a}\right) d a\right) d x \\
=\frac{b e^{r^{*} t}}{K b^{*}} \frac{R_{0}^{*}}{R_{0}} \int_{0}^{\beta}\left(e^{-\left(r-r^{*}\right) x}\right)\left(\int_{x}^{\beta} e^{-\left(r^{*}-k\right) a} m(a) p(a) d a\right) d x \\
=Y \int_{0}^{\beta}\left(e^{-\left(r-r^{*}\right) x}\right) \psi(x) d x
\end{gathered}
$$

Where, $Y=\frac{b e^{r^{*} t}}{K b^{*}} \frac{R_{0}^{*}}{R_{0}}$ and

$$
\psi(x)=\int_{x}^{\beta} e^{-\left(r^{*}-k\right) a} m(a) p(a) d a
$$

After solving the equation (36), we get

$$
\frac{Y}{\left(r-r^{*}\right)}\left[e^{k t} \frac{R_{0}}{R_{0}^{*}}-e^{k T}\right]=\frac{Y e^{k T}}{\left(r-r^{*}\right)}\left[\frac{R_{0}}{R_{0}^{*}}-1\right]=\frac{b e^{r^{*} t+k T}}{K b^{*}\left(r-r^{*}\right)}\left[1-\frac{R_{0}}{R_{0}^{*}}\right]=Z(\text { say })
$$

i.e. population at time $t$ is $Z$ times of the initial population.

If $r^{*}=0$ (i.e.for the stationary state), then $b^{*}=\frac{1}{e_{0}^{0}}, K \rightarrow \mu$ and $R_{0}^{*}=1$, (see Coale [1])

And the ultimate population size becomes

$$
\frac{b e_{0}^{0} e^{k T}}{\mu r}\left(1-\frac{1}{R_{0}}\right)
$$

(Model M6)

A more simplified case of model M6 may be by putting

$$
R_{0} \cong b e_{0}^{0} \text { (Fraunthal [3]) }
$$

and

$$
R_{0} \cong e^{r T} \cong e^{r \mu} \quad(\text { Coale [1]) }
$$

Into (42) and hence an ultimate population size as 


$$
\begin{array}{r}
\frac{e^{k T}}{\mu r}\left(R_{0}-1\right) \cong \frac{e^{k T}}{\mu r}\left(e^{r \mu}-1\right) \cong \frac{e^{k T}}{\mu r}\left(1+r \mu+\frac{r^{2} \mu^{2}}{2 !}+\ldots \ldots \ldots-1\right) \\
\cong e^{k T} e^{r \mu / 2} \cong e^{k T} R_{0}^{1 / 2}
\end{array}
$$

(Model M7)

Further, if $\mathrm{k}$ is small enough to put it zero, then the ultimate population is just $R_{0}^{1 / 2}$ times the initial population, which is given by Singh et.al. [13].

This shows that if only the net reproduction rate of a country/nation is known its ultimate population size can be worked out.

Thus several models proposed under the alternative fertility reduction are the extended form of the previous researchers like Keyfitz [4], Kumar [6], Kulkarni [7], Mitra [9] and many others.

\section{Data and Methodologies}

To obtain the size of ultimate population by the various models have developed here, we need the values of the demographic parameters $\mathrm{b}$ (birth rate of the initial stable population), $r$ (growth rate) and $e_{0}^{0}$ (expectation of life at birth for females). For illustration, the values of these parameters are taken from the "Regional Model Life Tables and Stable Population" of Coale and Demeney [2], a table consistent with the Indian situation and these are 23.8, 0.016 and 65.3 years (for females) respectively. The values of the other parameters are calculated from the maternity function $\varphi(x)=$ $p(x) m(x)$, based on data given in Appendix A. These are $R_{0}=1.546, \mu=28, \sigma^{2}=50.06$ and $K=28.19$.

\begin{tabular}{|c|c|c|c|c|c|c|c|c|c|c|c|c|c|}
\hline \multirow{4}{*}{$\begin{array}{c}\text { Time } \\
\text { A (in years) }\end{array}$} & \multicolumn{13}{|c|}{ Population Size ${ }^{*}$} \\
\hline & & & & & & & & \multicolumn{6}{|c|}{ Proposed Models } \\
\hline & \multirow{2}{*}{ Coale [1] } & \multirow{2}{*}{$\begin{array}{c}\text { Ryder } \\
{[11]}\end{array}$} & \multicolumn{2}{|c|}{ Kulkarni [7] } & \multicolumn{2}{|c|}{ Kumar [6] } & \multirow{2}{*}{$\begin{array}{c}\text { Keyfitz } \\
{[4]}\end{array}$} & \multirow{2}{*}{$\mathrm{M}_{11}$} & \multirow{2}{*}{ M2 } & \multirow{2}{*}{ M3 } & \multirow{2}{*}{ M5 } & \multirow{2}{*}{ M6 } & \multirow{2}{*}{$\mathrm{M}_{9}$} \\
\hline & & & Linear & Expo & Linear & Expo & & & & & & & \\
\hline 5 & 1.229 & 1.083 & 1.084 & 1.065 & 1.071 & 1.042 & \multirow{3}{*}{1.046} & 1.062 & 1.081 & 1.080 & \multirow{3}{*}{1.173} & \multirow{3}{*}{1.069} & \multirow{3}{*}{1.2794} \\
\hline 10 & 1.179 & 1.130 & 1.132 & 1.074 & 1.117 & 1.078 & & 1.173 & 1.130 & 1.175 & & & \\
\hline 15 & 1.226 & 1.179 & 1.183 & 1.173 & 1.154 & 1.180 & & 1.133 & 1.165 & 1.135 & & & \\
\hline
\end{tabular}

Table 1. Ultimate Population Size for Different values of a Under Various Models of fertility Reduction

* times the initial population. Assuming initial population=1

Source: (i) Regional Model Life Tables and Stable Populations (Female South Level), Coale and Demeny. 1966 (ii) Shruti (2002), Some Population Growth Models and Their Applications, Unpublished Ph.D. Thesis in Statistics, BHU, Varanasi, India

Table 2. Ultimate Population Size for Different values of $A_{1}$ and $A_{2}$ Under model M1

\begin{tabular}{|c|c|c|c|}
\hline $\mathrm{A}_{2}$ (in years) & \multicolumn{3}{|c|}{ Population Size* } \\
\hline $\mathrm{A}_{1}$ (in years) & 0 & 5 & 10 \\
\hline 5 & 1.043 & 1.040 & \\
\hline 10 & 1.110 & 1.062 & 1.094 \\
\hline 15 & 1.134 & & \\
\hline
\end{tabular}

*times the initial population. Assuming initial Population=1

Source: (i) Regional Model Life Tables and Stable Populations (Female South Level), Coale and Demeny. 1966

(ii) Shruti (2002), Some Population Growth Models and Their Applications, Unpublished Ph.D. Thesis in Statistics, BHU, Varanasi, India

\section{Illustration and Discussions}

Table 1 presents the ultimate size of the population under various models for India upto the lower limit of the reproductive period. Moreover, the ultimate population size estimated under various proposed models has been compared with the ultimate population size obtained by the other researchers including the model of Keyfitz [4]. Table 2 gives the projected population for different values of $A_{1}$ and $A_{2}\left(A_{1}<A_{2} \leq \alpha\right)$ by model M1. For example, if $A_{1}=5$ years and $A_{2}=10$ years, the ultimate population size by the Model came out to be 1.134 times the initial population (see also Figure 3 ). This figure shows that a linear change in fertility for a longer time yield lesser size of ultimate population than exponential change. 


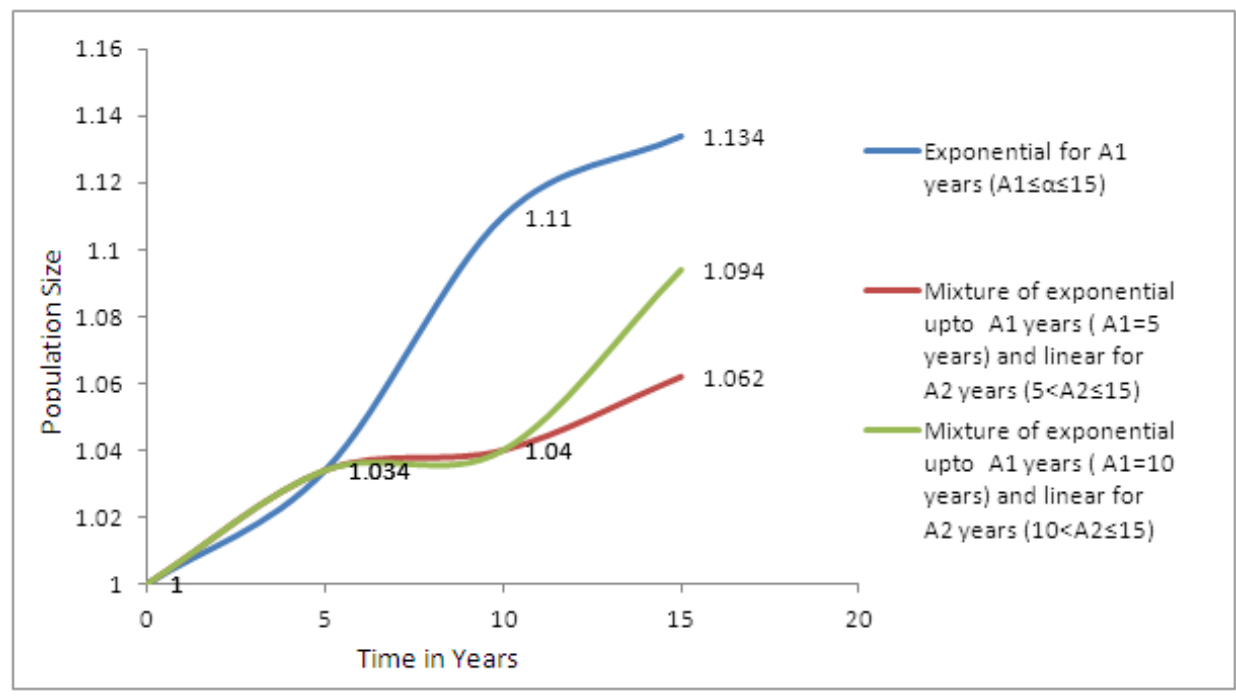

Figure 3. Showing the size of ultimate population under various scheme of change in fertility schedule

Similarly if we take the value of $\mathrm{k}=0.001$, the ultimate population size from model M6 was found to be 1.069 times the initial population. The ultimate size of population by models M5 and M7 was 1.414 and 1.083 times the initial population respectively (see also Figure 4). This figure 4 also shows that the ultimate population size obtained by the proposed models provide more or less similar results provided by the models developed by the previous researchers. An upward bias of about 27 and 4 per-cent was found involved in the size of the population under the simplest models M5 and M7 respectively. Thus the models M5 and M7 would be useful for the projection of population size where reliable and accurate data on demographic parameters are not available. However, the validity of a population model depends on the way of increase or decrease of the net maternity function $(p(x) m(x))$.

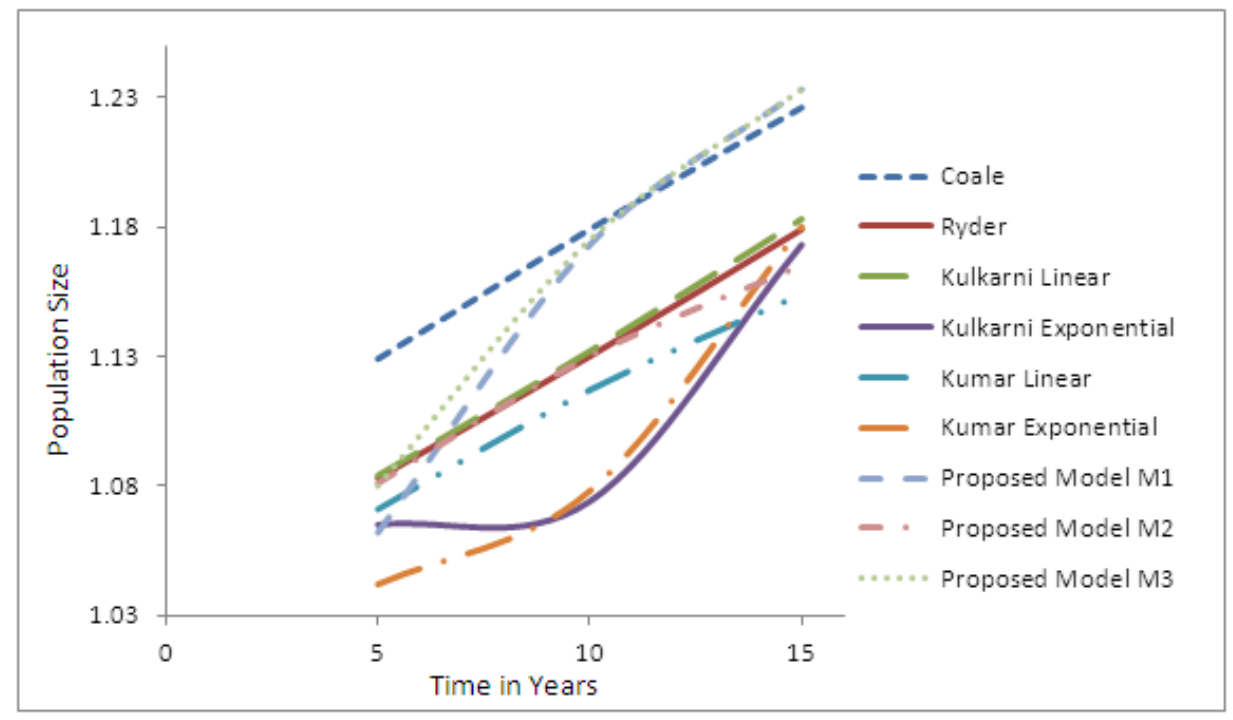

Figure 4. Showing the size of ultimate population under various scheme of change in fertility Schedule by the proposed model along with models given by the several authors

Nevertheless, the reduction in fertility schedule from $m(x)$ to $e^{-r x} m(x)$ seems to be more reasonable as it depends on the age of females in the reproductive period in comparison to reduction like $m(x)=m(x) R_{0}$ (Keyfitz, 1971). Further, an abrupt change in fertility (as shown by Figure 2) may not yield the expected size due to momentum created by a high force of fertility build up in the past (Keyfitz [5]). 


\section{Conclusions}

To obtain the size of an ultimate population under the stability conditions, a number of models have been developed under the various paths of fertility reduction. A model for the ultimate population size is also developed making use of the reproductive value as well as taking an increase in the survival function. Taking some hypothetical data consistent with the Indian demographic scenario, the models have been illustrated where possible explanations are given regarding merits and demerits of a model. Some models have also been approximated to extent of minimum number of demographic parameters to predict the size of ultimate population. These models are useful for many underdeveloped and developing countries where demographic data are found unreliable or inaccurate.

It has also been found that models derived in this paper are the generalization of all most all the previous models.

\section{Acknowledgements}

The authors are grateful to the referee for providing valuable comments on the original drafts of the paper to improve its quality.

\section{Appendix A}

Values of Age Specific Fertility Rate (ASFR) and m(x) Function

\begin{tabular}{|c|c|c|c|}
\hline Age Group & ASFR & $\begin{array}{c}\text { Proportion of eligible } \\
\text { females (couples) }\end{array}$ & $\mathrm{m}(\mathrm{x})$ \\
\hline $15-19$ & 0.116 & 0.723 & 0.856 \\
\hline $20-24$ & 0.231 & 0.959 & 0.846 \\
\hline $25-29$ & 0.170 & 0.944 & 0.834 \\
\hline $30-34$ & 0.097 & 0.984 & 0.822 \\
\hline $35-39$ & 0.044 & 0.866 & 0.809 \\
\hline $40-44$ & 0.015 & 0.849 & 0.796 \\
\hline $45-49$ & 0.005 & 0.823 & 0.779 \\
\hline
\end{tabular}

Source: Shruti (2000) Some Population Growth Models and Their Applications, Unpublished Ph.D. Thesis in Statistics, BHU, Varanasi, India Computation of $\mathbf{m}(\mathbf{x})$

If the age specific fertility rate (ASFR) for female births only is known then it is needed to convert it into age specific marital fertility rate (ASMFR).Since ASFR includes all the females and this does not take into account whether a female is eligible for conception or not. Thus the values of ASFR are divided by the proportion of eligible couples of a particular group for finding the values of ASMFR. Once the values of ASMFR are known, $\mathrm{m}(\mathrm{x})$ can be calculated as: $\mathrm{m}(\mathrm{x})=\mathrm{ASMFRxQx} 5$

where $\mathrm{Q}$ is the sex ratio at birth. Multiplication by 5 comes due to 5 yearly group of the females (couples).

\section{REFERENCES}

[1] Coale,A.J. (1972), "The Growth and Structure of Human Populations : A Mathematical Investigation", Princeton University Press, Princeton, New Jersey, USA.

[2] Coal,A.J. and Demeny,P., (1966), "Regional Model Life Table and Stable Population", Princeton University Press, Princeton, New Jersey, USA.

[3] Frauenthal, J.C. (1975),"Birth Trajectory Under Changing Conditions" Demography, 12, 447-454

[4] Keyfitz,N. (1971), "On the Momentum of Population Growth", Demography, Vol. 8, pp. 71-80.

[5] Keyfitz,N. (1977)," Applied Mathematical Demography", John Wiley \& Sons, New York

[6] Kumar,U. (1991), "Some Models for Migration and Population Growth", Unpublished Ph.D. Thesis, BHU, Varanasi, India.

[7] Kulkarni,P.M. (1976), "Growth of Population After Reduction in Fertility", Newsletter 11(1), Population Center Bangalore, India.

[8] Lotka,A.J. (1939), "Theories analytique des associations bio-logiques Part II. Analyse demographique avee application particuliere a L' Espece Humaine", Actualities Scientifiques et Industrielles, No. 780, Paris: Herman and Cie.

[9] Mitra,S. (1976), "Influence of Instantaneous Fertility Decline to Replacement Level on Population Growth : An Alternative Models", Demography, Vol.13, pp. 513-519.

[10] Pathak,K.B. and Ram,F. (1985), "Trends in Reproductive Values of the Females and Momentum of Population Growth : Analysis for the Major States of India ", IIPS, Mumbai, India.

[11] Ryder,N.B. (1975), "Notes on stationary population", Population Index, Vol. 41(1), pp.3-28.

[12] Singh,S.N., Yadava,R.C. and Yadava,K.N.S. (1981a), “A Mutiregional Generalization of Keyfitz Momentum Formula Under a Less Drastic Fall in Fertility", The Aligarh Journal of Statistics, Vol. 19 (1), pp. 59-68.

[13] Singh,S.N., Yadava,R.C. and Yadava,K.N.S. (1981b), “A Simplified from of Keyfitz Momentum Formula Under a Less Drastic Fall of Fertility", Journal of Indian Statistical Association, Vol.19, pp.171-176.

[14] Yadava, K.N.S. (1985),'Population Projection upto the Lower Limit of the Reproductive Period", Demography India, $15,146-150$.

[15] Yadava,K.N.S., Kumar,U. and Kushwaha,S.N.S. (1989), "Population Projection upto the Lower Limit of Reproductive Period Under Gradual Change in Fertility Schedule : Some Alternative Models", Demography India, Vol. 18, pp. 73-80.

[16] Yadava,K.N.S., Yadava,S.N. and Sarin,D. (1996), "Population Growth Under Changed Fertility Schedule in Stablity Conditions", Mathematical Population Studies, Vol. 6(1), pp. 55-65.

[17] Yadava, R.C., Yadava, K.N.S. and Singh, V.S. (1998),"Population Projection under a Changed Regime of Fertility", Demography India, 27, 275-286. 